\title{
Real-Time Dynamic Planning to Maintain Network Connectivity in a Team of Unmanned Air Vehicles
}

\author{
Andrew N. Kopeikin ${ }^{1}$, Sameera S. Ponda ${ }^{1}$, Luke B. Johnson ${ }^{1}$, Olivier Toupet ${ }^{2}$, Jonathan P. How ${ }^{3}$ \\ ${ }^{1}$ Department of Aeronautics \& Astronautics, Massachusetts Institute of Technology, MA 02139 USA \\ ${ }^{2}$ Aurora Flight Sciences Corporation, Cambridge MA 02139 USA \\ ${ }^{3}$ R. C. Maclaurin Professor of Aeronautics and Astronautics, Massachusetts Institute of Technology, MA 02139 USA
}

\begin{abstract}
As technology progresses, the capability provided by teams of unmanned systems will span all operational domains and become increasingly important in many applications. Teams of unmanned systems can be composed of agents with different capabilities, and must often operate in dynamic environments where the state of tasks is always changing. Effective real-time task allocation is paramount in these situations. Constraints on connectivity of a network of unmanned agents, such as those required to provide realtime remotely sensed data for exploitation, are a major challenge in this planning process. This paper presents the implementation of research performed to extend a real-time task planning approach called the Consensus Based Bundle Algorithm (CBBA), to include planning for communication relays when facing potential network disconnects. The paper implements the algorithm in a flight test experiment with a team of 3 UAVs performing observation tasks out of communication range from the base station. Results show that planning for relays can be performed in real-time and offers increased mission performance over the baseline CBBA algorithm.
\end{abstract}

Index Terms - teams of unmanned air vehicles, cooperative control, communication relays, network connectivity.

\section{INTRODUCTION}

$\mathrm{A}^{\mathrm{s}}$ technology keeps improving, teams of unmanned systems will play an increasingly important role in a broader range of applications. These teams may span across different operational domains such as air, ground, surface, underwater, and space, and may be used in both civilian and military applications [1]-[3]. The "real-world" situations which utilize teams of unmanned systems will involve executing complex missions where the number, status, and types of tasks, as well as the environment, vary dynamically. Proper planning in unmanned agent resource allocation is therefore critical to ensure that tasks are performed effectively and efficiently, and to maximize the overall mission performance of the system.

A significant challenge common in many unmanned system operations lies in maintaining proper communications to execute the mission. In most circumstances, a communications link is required between the robot in operation and the base station where commands are issued and data remotely sensed is processed and utilized [1]. The lack of communication among agents in teams of unmanned systems can create difficulties in mission planning [4,5]. Wireless communications can be disconnected because of obstacles to line-of-sight, ranges beyond the capability of the equipment, and interference from a variety of sources [6].

One approach to preventing network disconnects in a team of unmanned assets is to employ members of the team as communication relays where and when it is necessary to transfer information from out-of-reach robots to the base. Several authors describe methods to plan for relays, but these involve complex algorithms which scale poorly as the number

Manuscript received September 3, 2011.

Corresponding author: A. N. Kopeikin (e-mail: kopeikin@mit.edu). of agents, tasks, and constraints increase [7,8]. Such methods are therefore difficult to apply to real-world situations.

In order to plan for communication relays, communication constraints must be considered in the task allocation. This can be a difficult problem due to high computational cost and changing dynamic constraints. In this paper, we extend recent work on a real-time task allocation framework which effectively plans for communication relays as needed to repair disconnects in the network, and implement it in flight test. In the framework, agents receive the task locations and information to make their initial plans, predict the state of the network, detect potential disconnects, and, if possible, create relay tasks that will enable them to maintain connectivity to the base station. At this stage, the unmanned agents can reevaluate their decisions based on the new relay tasks created, and decide to take on a relay role to achieve greater overall mission performance. Past results have shown, in a controlled setting, that using certain systems as communications relays can increase mission performance for a team of unmanned ground vehicles.

The paper is organized as follows. Section II provides the initial formulation for the multi-agent multi-task allocation framework. Section III describes the extension of the task allocation algorithm which maintains network connectivity through the use of relays. Section IV discusses the flight test implementation and results, as well as a recent extension to the algorithm.

\section{UnMANNED System TEAm TASK Allocation}

\section{A. Problem Formulation}

The task allocation problem involves planning the resource allocation in a team of $N_{a}$ unmanned systems, to accomplish a set of $N_{t}$ tasks which can each have time-windows of validity. The goal is to maximize reward for the mission by assigning 
the right asset, to the right task, at the right time, and avoid conflicts of assigning any one task to more than one agent. The task assignment is formulated as follows [9]:

$$
\max \sum_{i=1}^{N_{a}}\left(\sum_{j=1}^{N_{t}} c_{i j}\left(\mathrm{p}_{i}\left(\mathrm{x}_{i}, \tau_{i}\right)\right) x_{i j}\right)
$$

subject to: $\sum_{j=1}^{N_{t}} x_{i j} \leq L_{t}, \forall i \in \mathcal{J}$

$$
\begin{aligned}
& \sum_{i=1}^{N_{a}} x_{i j} \leq 1, \forall j \in \mathcal{J} \\
& x_{i j} \in\{0,1\}, \tau_{i j} \in\left\{\mathbb{R}^{+} \cup \emptyset\right\}, \forall(i, j) \in \mathcal{J} \times \mathcal{J}
\end{aligned}
$$

here $x_{i j}=1$ if agent $i$ is assigned to task $\mathrm{j}, x_{i}$ is its vector of assignments, and $c_{i j}$ is the score it receives for doing each task. Vector $\mathrm{p}_{i}$ represents the path for agent $i$. The vector of times $\tau_{i}$ is the times that agent $i$ proposes to perform the tasks in the path. A maximum of $L_{t}$ tasks can be assigned to each agent, and the maximum overall number of assignments achievable is given by $N_{\max } \triangleq \min \left\{N_{t}, N_{a}, L_{t}\right\}$ [9]. The objective function is the sum of reward values for each agent $i$, while each reward is determined as a function of the tasks assigned to the particular agent.

\section{B. Consensus-Based Bundle Algorithm (CBBA)}

Because of all inter-dependencies in number of possible assignments, Equation (1) can be a very difficult problem to solve if planning for more than 1 task for each agent. A popular method to solve the task assignment problem involves using auction algorithms which are efficient but sub-optimal $[10,11]$. The Consensus-Based Bundle Algorithm (CBBA) is one such auction protocol that provides provably good solutions for heterogeneous decentralized agent multi-task assignment functions. Each CBBA iteration is composed of two phases. First, in the bundle building phase each agent greedily bids on an ordered bundle of tasks based on its capabilities and its location with respect to the task. Second, in the consensus phase, agent bid information is shared, and conflicts in task assignment are identified and resolved. The algorithm iterates until it converges on a conflict free plan [12]. The CBBA algorithm runs in polynomial time, and has demonstrated good scalability with increasing numbers of agents and tasks. It has been demonstrated in numerous applications with teams of heterogeneous unmanned systems [13].

\section{CBBA WITH COMMUNICATION RELAYS}

In order to execute CBBA task assignment, agents must be able to communicate with the base station and with each other. The performance of each communication link can be modeled according to different parameters. In the experiment presented in this paper, we consider two agents to be connected if they are within a prescribed communication radius of each other, based on Euclidean distance. Later we present some initial work to study planning for connectivity when nodes face lineof-sight constraints. Agents are considered to be connected to the network as long as they are connected through at least one link to the base station. If all agents are connected to the network and can share bid information, CBBA ensures a conflict free task assignment. In addition, an agent connected to the network can provide reward to the mission by performing that task and sending the collected data to the base for processing and exploitation. Disconnected tasks should be avoided since they provide no reward but waste energy in their execution.

The objective of this research is to implement and flight test a method developed to identify potential disconnects in the team, and plan for under-utilized members to serve as relays to strengthen connectivity. Here the baseline CBBA task assignment algorithm is initialized with an empty set of assignment vectors, a relay list, and a forbidden task list for each agent [9]. CBBA is executed after which the network computes the predicted network topology and identifies any possible disconnects. To ensure network connectivity, two different approaches can be taken at this point. First, a conservative approach is to simply drop any tasks that result in a network disconnect. While this method does ensure network connectivity, it can severely limit the mission performance of the system by favoring tasks that are closer to the base station. In order to have more flexibility in achievable tasks, the CBBA with Relays approach (Figure 1) was proposed [9].

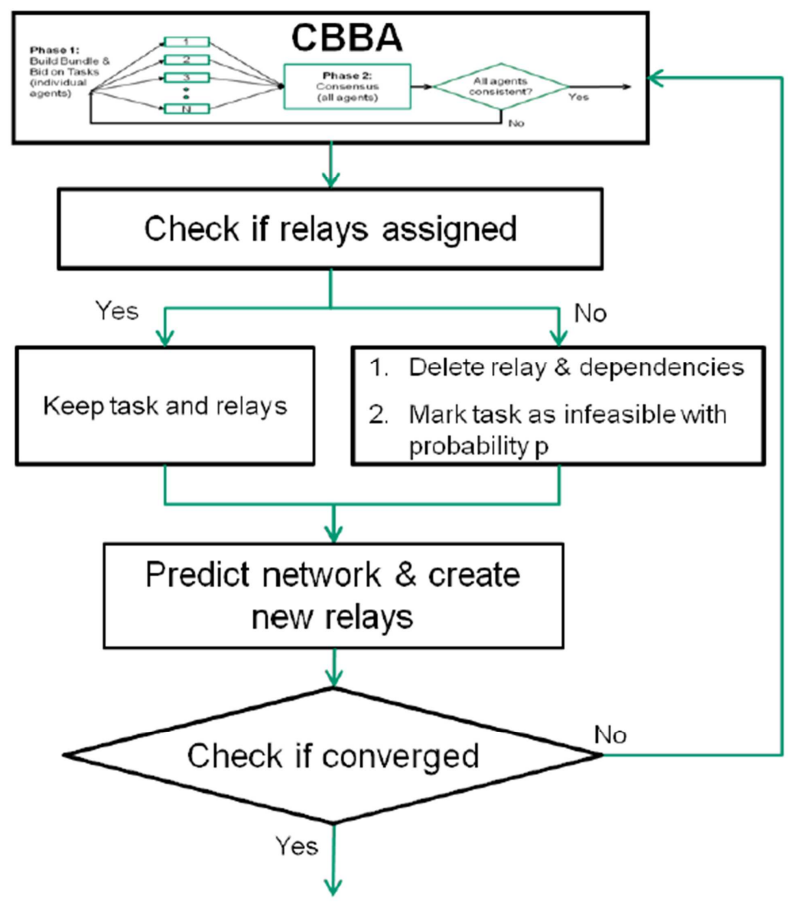

Figure 1. Block diagram of the CBBA with Communication Relays algorithm

Here, when potential disconnects are identified in the task planning algorithm, relay tasks are created to repair the link. The CBBA algorithm now iterates again, with the relay tasks. If agents can accommodate relay tasks into their plan they place an appropriate bid on the task. Following each iteration of the algorithm, unassigned relays are deleted. The tasks dependent on the deleted relays are then placed in the bidding 
agent's forbidden task list. This ensures that the CBBA with Relays algorithm eventually converges on a task assignment solution. Figure 1 shows a block diagram of the CBBA with Relays algorithm.

\section{FIELD EXPERIMENT}

\section{A. Implementation and Setup}

Previous work has included an experiment using the CBBA with Relays algorithm conducted for a team of unmanned ground vehicles in the controlled setting of the MIT Real-time indoor Autonomous Vehicle test ENvironment (RAVEN) [9]. In order to expand the application of the algorithm and demonstrate it in a more operationally realistic environment a field experiment was designed to show the capability of CBBA with Relays in outdoor flight testing. The mission scenario consisted of gathering information in the environment using small research grade UAVs. The communication radius $\left(\mathrm{R}_{\mathrm{COMM}}\right)$ of any one UAV was set to $30 \%$ of the flight arena length. In the scenario, an area of greater information interest was designated at distances greater than $\mathrm{R}_{\text {СOMM }}$ from the base station, and occupied half of the flight arena (Figure 2 (a)). Information gathering tasks were then randomly created with uniform distribution across the flight arena. Each task consisted of a location, altitude, and time window for a UAV to fly to and take an observation. Tasks inside the area of greater interest were set to yield between 7.5 and 10 times more reward than tasks outside that area, based on the distance from the base. This incentivized execution of these tasks even though they required a relay to stay connected.

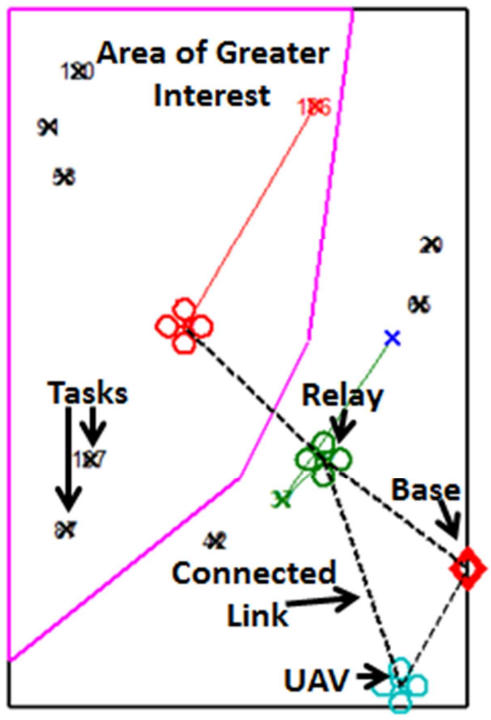

(a) Mission setup \& execution
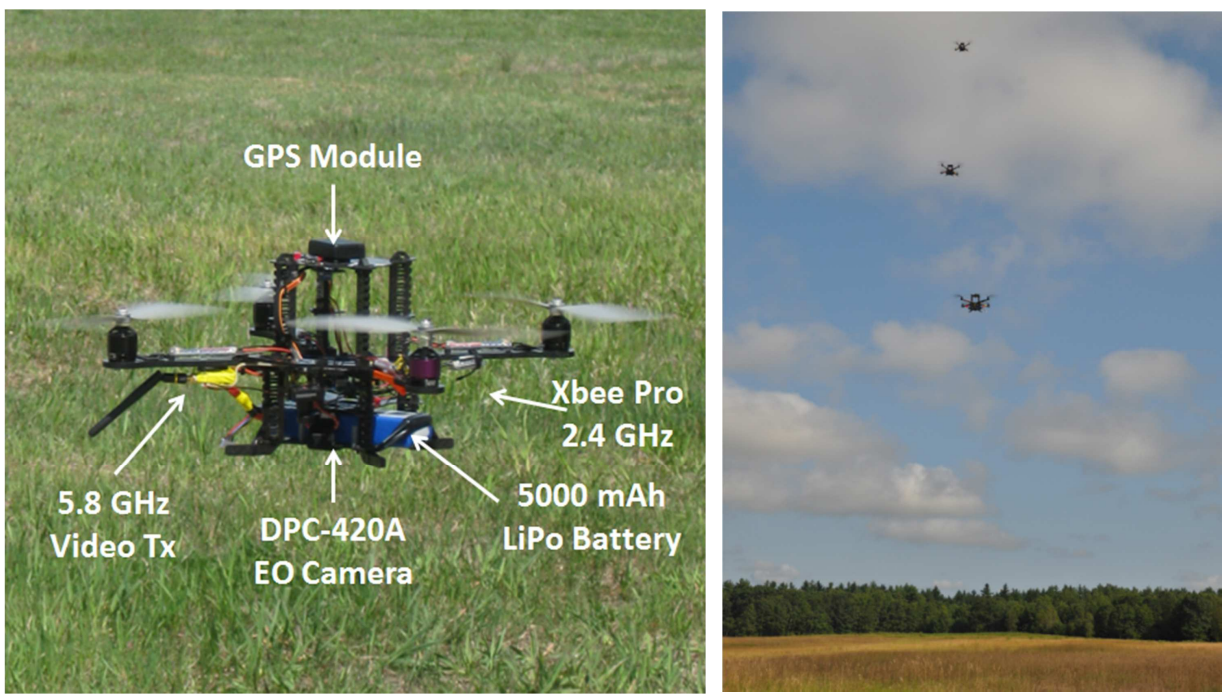

(b) Ascending Technologies Pelican Quadrotor

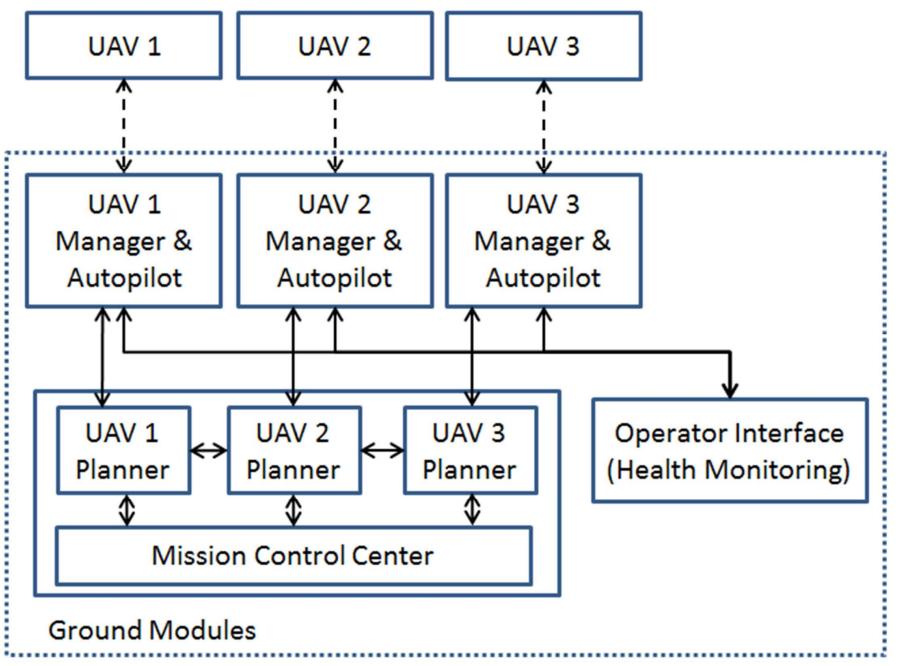

(c) Implementation system architecture

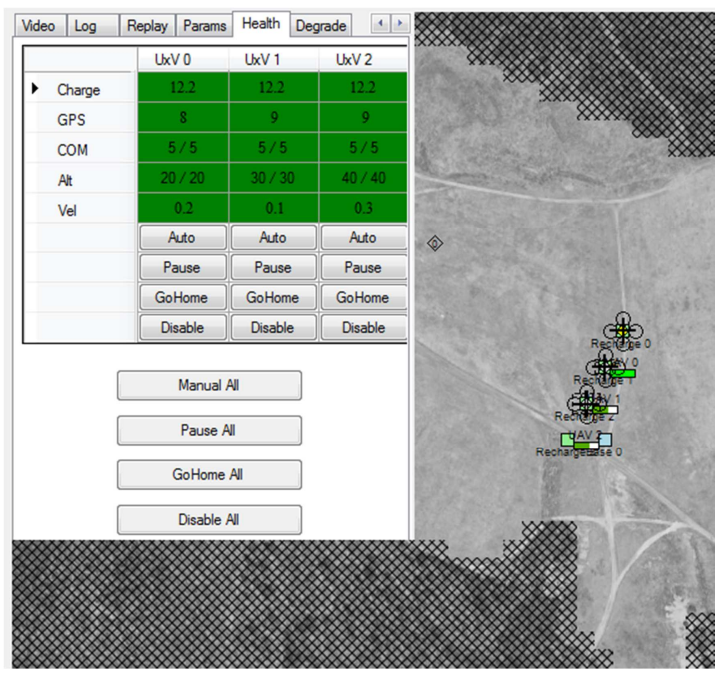

(d) Operator Interface with health monitoring panel

Figure 2. Field experiment implementation elements 
The UAVs used in this experiment consisted of 3 Ascending Technologies Pelican Quadrotors shown in Figure 2 (b). Each vehicle is $2.5 \mathrm{lbs}$, has a flight endurance of 18 minutes, and is capable of GPS waypoint navigation while communicating with the Ground Control Station (GCS) using a Digi-Mesh XBee $2.4 \mathrm{GHz}$ radio module. The $3 \mathrm{UAV}$ distributed system was in part integrated with the Aurora Flight Sciences multivehicle control framework which was recently developed and demonstrated to control 4 heterogeneous UAVs executing a complex mission using CBBA planning under human supervision [13]. Here, the system architecture seen in Figure 2 (c) consisted of several software modules communicating via the Lightweight Communications and Marshaling (LCM) UDP multicast protocol [14]. First the MATLAB Mission Control Center created the set of random tasks in the mission, and the Task Allocation Planner executed the distributed planning for each UAV agent. The assignments were then communicated to the $\mathrm{C}++$ based vehicle management and autopilot modules to compute the flight path and communicate waypoints to the vehicles. A C\# developed Operator Interface was used for a human operator to oversee the mission execution and monitor the health and status of the UAVs during test and evaluation (Figure $2(d)$ ). Each vehicle was assigned a safety pilot who remained in radio contact with the GCS operator, and had the ability to regain manual control of the UAV in the case of an abnormal situation. All flights where conducted at Fort Devens, MA in restricted airspace.

\section{B. Flight Test Experiment Results}

Three sets of missions were executed, each using different planning strategies: (1) baseline CBBA with no consideration for network connectivity, (2) an approach where tasks which create disconnects are dropped from the plan (named CBBA with Network Prediction), and (3) CBBA with Relays. The missions were first performed in software simulation, and were then executed in outdoor flight tests. Figures 3 and 4 show the results of the experiment. The mission score plot is a reflection of the cumulative reward as the mission progresses. The reward varies as tasks are executed and fuel is consumed traveling to the task locations. The plots show that there is stronger connectivity when using CBBA with Relays and Network Prediction than when planning with the baseline algorithm. CBBA with Relays has the highest performance when comparing mission scores since agents collaborate to accomplish valuable tasks in the search area and relay the information to the GCS. The baseline CBBA strategy has the lowest performance since agents consume energy to travel to tasks for which they receive no reward.

The plots also indicate that the simulation results follow the same trends as the hardware results. This demonstrates that the algorithm is capable of operating during the mission execution and can overcome elements of communication drop outs and erroneous state estimates from the vehicles flying outdoors. Two effects were observed in the field which had not been captured in simulation. First, each replan takes time (up to 20 seconds in some instances). In simulation, time stops during replans and therefore the vehicle positions remain the same as the plan converges. During flight tests, vehicles continued to navigate during the planning phase, and could therefore reach a configuration making introducing flaws into the plan by the time it converged. Second, the actual velocities of the vehicles differ from what was predicted in planning. Therefore UAVs can reach task locations and start executing them at times different than what was planned for (by only a few seconds). Again this could result in a flawed plan. The combination of these two effects explains why there was one disconnected task witnessed using CBBA with Relays in flight testing. These lessons learned should be incorporated into the simulation for future testing. Overall six successful sorties involving three quadrotors where executed to successfully integrate and collect data in this experiment.

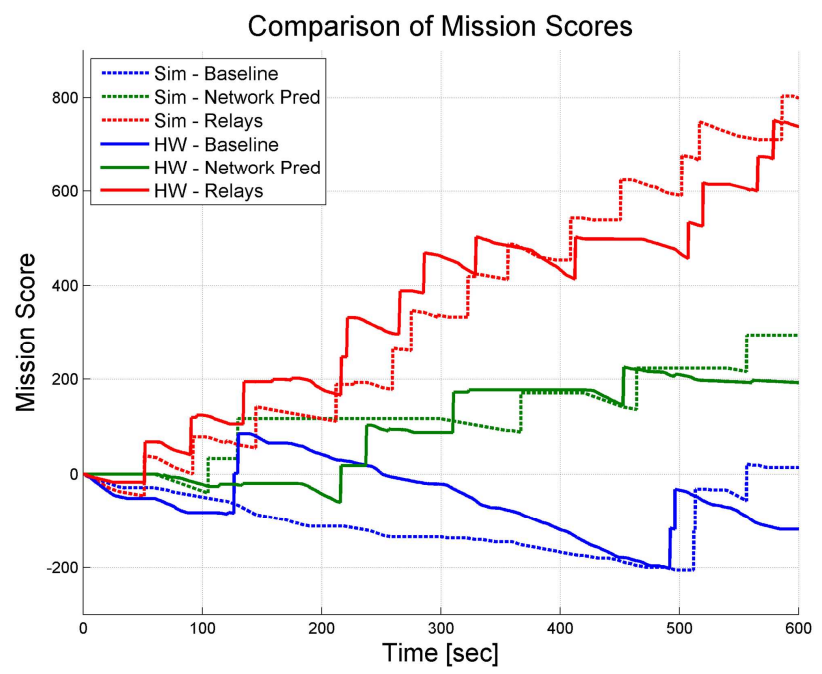

Figure 3. Comparison of field test mission scores

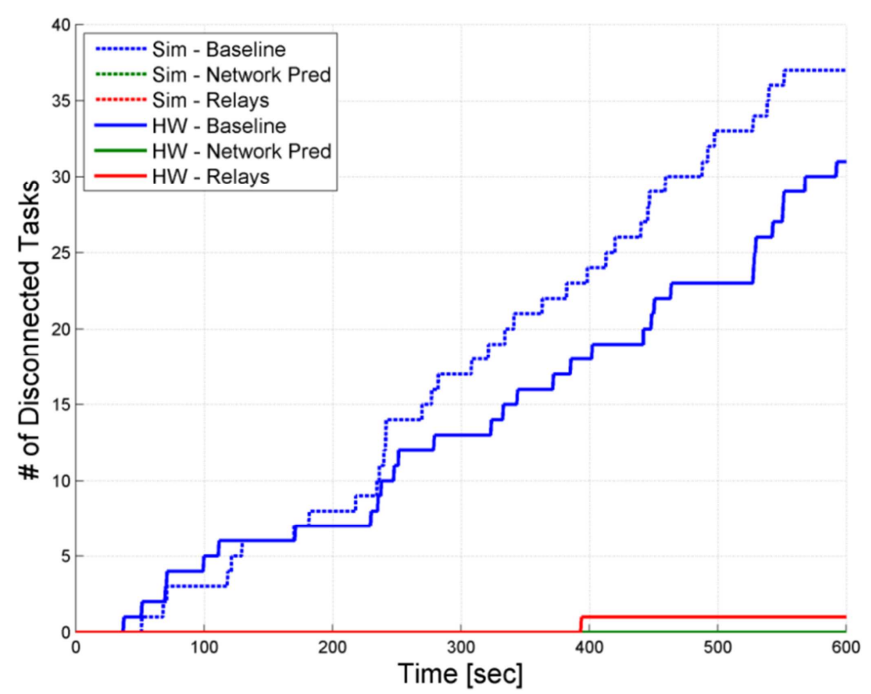

Figure 4. Comparison of field test disconnected tasks

\section{Line-of-Sight Constraints Simulation Results}

In addition to the field implementation, the planning 
algorithm was extended to relay tasks disconnected from the network because of a vertical obstruction to Line-of-Sight (LOS) such as a wall or mountain. Here the planner places a relay at the minimum Euclidean distances between the two nodes to be connected with LOS over the top of the obstruction. A maximum allowable altitude for the UAVs may be established because of aircraft performance or airspace limitations. The algorithm positions the relay at the optimal location meeting that restriction (Figure 5). An experiment was conducted in simulation using the previously described scenario for 10 minute missions. Here the area of greater interest was separated from the base by a $35 \mathrm{~m}$ tall ridge, all tasks were to be executed at a height of $20 \mathrm{~m}$, and the maximum allowable altitude was $50 \mathrm{~m}$. The results show increased mission performance when planning for relays and the lowest performance when using the baseline algorithm (Figure 6). Tasks were only disconnected when using baseline CBBA.

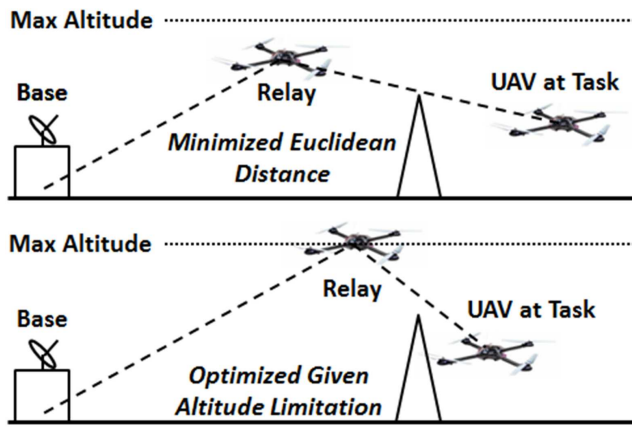

Figure 5. Planning relay over obstruction to LOS

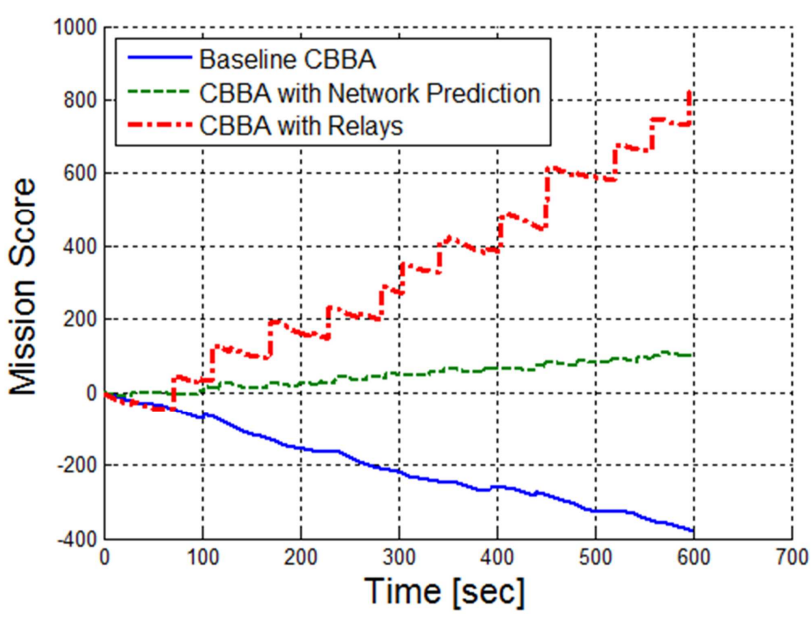

Figure 6. Simulation results with LOS constraints

\section{CONCLUSIONS}

This paper presented outdoor flight tests to perform realtime dynamic task allocation while planning to maintain network connectivity for a team of UAVs. An extension of the CBBA algorithm assigns under-utilized agents to serve as communication relays for others to execute tasks which would otherwise be disconnected. A framework was implemented where 3 quadrotors flew outdoors executing information gathering tasks, often beyond the communication radius of any one UAV. Results show improved mission performance when planning with relays. In addition, the algorithm was extended to plan relays for agents disconnected because of a vertical obstruction.

Future work will extend the algorithm so that one agent can simultaneously perform multiple relay tasks. The Line-ofSight formulation will be also refined to handle more complex scenarios, and Graph Laplacians and control algorithms for ad-hoc networks will be investigated to improve connectivity $[6,15]$.

\section{ACKNOWLEDGMENTS}

Authors thank MIT students Aaron Prindle and Paul Quimby for their support during the flight tests. Work sponsored (in part) by the AFOSR and USAF under grant FA9550-08-10086, ONR STTR N00014-08-C-0707, and MURI (FA955008-1-0356). The views and conclusions contained herein are those of the authors and should not be interpreted as representing the official policies or endorsements, either expressed or implied, of the U.S. Government.

\section{REFERENCES}

[1] “Unmanned aircraft systems roadmap, 2005-2030". Tech. rep., Office of the Secretary of Defense.

[2] C. Marshall, M. Mears, S. Rasmussen, "2010 ICE-T cooperative control flight testing," AIAA Aerospace Information Technologies Conference, St Louis, MS. 2011.

[3] N. E. Leornard, D. A. Paley, R. E. Davis, D. M. Fratantoni, F. Leklen, F. Zang. "Coordinated control of an underwater glider fleet in an adaptive ocean field experiment in Monterey Bay". J of Field Robotics 27(6). 718-740.

[4] R. Olfati-Saver, R. M. Murray, "Consensus problems in networks of agents with switching topology and time-delays", IEEE Trans. Automat. Contr. Vol. 49, no. 9, 2004, pp1520-1533.

[5] S. Ponda, J. Redding, H.L. Choi, J.P. How, M.A. Vavrina, and J. Vian, "Decentralized planning for complex missions with dynamic communications constraints", American Control Conference, 2010.

[6] P. Santi. "Topology control in wireless ad hoc and sensor networks", ACM Computing Surveys, Vol. 37, no. 2, 2006, pp. 164-194.

[7] H. Nguyen, N. Pezeshkian, M. Raymond, A. Gupta, and J. Spector, "Autonomous communication relays for tactical robots", Proceedings of the International Conference on Advanced Robotics (ICAR), 2003.

[8] A. Ibrahim, K. Seddik, K. Liu, "Improving connectivity via relays deployment in wireless sensor networks", Global Telecommunications Conference (GLOBECOM), 2007, pp. 1159-11.63.

[9] S. Ponda, L.B. Johnson, H.L. Choi, J.P. How. "Ensuring network connectivity for decentralized planning dynamic environments", AIAA Aerospace Information Technologies Conference, St Louis, MS. 2011.

[10] S. Sariel, and T. Balch, "Real time auction based allocation of tasks for multi-robot exploration problem in dynamic environments", AIAA Workshop on Integrating Planning Into Scheduling, 2005.

[11] A. Ahmed, A. Patel, T. Brown, M. Ham, M. Jang, G. Agha, "Task assignment for a physical agent team via a dynamic forward/reverse auction mechanism", International Conference on Integration of Knowledge Intensive Multi-Agent Systems, 2005.

[12] H.L. Choi, L. Brunet, and J.P. How, "Consensus-based decentralized auctions for robust task allocation", IEEE Trans. On Robotics, vol. 25 (4), 2009, pp. 912-926.

[13] A. Kopeikin, O. Toupet, A. Clare, J.P. How, M.L. Cummings "Design of a decentralized multi-UAV system with human supervision", AUVSI North America, Washington DC. 2011.

[14] A.S. Huang, E. Olson, D. Moore, "Lightweight communications and marshalling for low-latency interprocess communications," Computer Science and Artificial Intelligence Laboratory Technical Report, Massachusetts Institute of Technology, MA. 2009.

[15] R. Olfati-Saber, A. Fax, R.M. Murray, "Consensus and cooperation in networked multi-agent systems" Proceedings of the IEEE, vol. 95 (1), 2007 , pp. 215-233. 